\title{
Thin-Walled Tube Extension by Rigid Curved Punch
}

\author{
Rostislav I. Nepershin \\ Plastic Deformation Systems Department, Moscow State University of Technology "STANKIN", \\ Moscow, Russia \\ E-mail:nepershin_ri@rambler.ru \\ Received February 22, 2011; revised March 16, 2011; accepted March 31, 2011
}

\begin{abstract}
Computer model is developed for non-steady and steady-state process of thin-walled tube extension by the rigid punch with curved profile. Rigid-plastic membrane shell theory with quadratic yield criterion is used. Tube material normal anisotropy, work hardening, wall thickness variation and friction effects are considered. FORTRAN programs of the model predict distributions of the thickness, meridian stress, yield stress and pressure along curved generator of deformed tube and the tube extension force versus punch displacement relation. Model predictions are correlated with experimental data.
\end{abstract}

Keywords: Tube Extension, Rigid-Plastic Membrane Shell, Curved Rigid Punch, Normal Anisotropy, Work Hardening, Wall Thickness Variation

\section{Introduction}

Thin-walled tube extension by rigid punch with curved generator can be used for forming of specified profiles at the tube ends defined by its function in machine design. Approximate analysis of the thin-walled tube extension by rigid punch is constrained, mainly, by conical form with approximate yield criterion of isotropic material, approximate wall thickness variation and work hardening effect [1-4]. Practical technology of the tube extension by cone punch reveals curved generators forming at transition regions from cone to cylinder parts of the tube [2-4], which are difficult to control. But curved generator can be specified by hydro- or aerodynamic parameters of the tube profile. Thin-walled metal tube can reveals normal anisotropy induced by cold rolling technology with the result of stress-strain and wall thickness effects during tube extension. So, development of computer model of the tube extension process by rigid punch with curved generator and material normal anisotropy consideration, deems, is important engineering problem.

Simulation of non-steady tube plastic forming by the finite element method (FEM) is limited by difficult problem of large matrix Equations accurate solution for nonlinear plastic material model with work hardening effect, stress-strain relations, variable shell thickness and curved tool boundary. Nonlinear plastic problem in commercial FEM codes is treated as non-linear elasticity or viscous solid without finite yield stress, with the result of non-accurate stress state calculations. More accurate simulation of thin-walled tube plastic forming, deems, should be made by correct numerical solution of ordinary differential Equation derived from exact equilibrium Equations of membrane rigid-plastic thin-walled shell model with Mises yield criterion, including work hardening and normal anisotropy effects. This approach was used successfully for non-steady models of plastic shells drawing, which good correlated with experiments [5-8], and for thin-walled tube reduction by matrix with curved profiles [9].

Presented model of the thin-walled tube extension by rigid punch with curved generator is based on membrane theory of the rigid-plastic shell of revolution, with material normal anisotropy, work hardening, wall thickness variation and friction effects included. FORTRAN programs are written for numerical solution of the problem differential Equations. Numerical results of computer simulation are given for the $S$ - mode cosine, double circular and cone-circular punch profiles. Presented model for cosine punch profile and related model [9] for the tube reduction by curved matrix are reasonable correlated with experimental data.

\section{Problem Formulation}

Scheme of the thin-walled tube extension by the punch with curved generator is shown in Figure 1. Cylindrical co-ordinates $r, z, \theta$ are related with fixed punch, while 
the tube is moved in positive $z$ direction. The punch curved profile is specified by differentiable function $r=r$ (z) on the axial length $H_{0}$ with continues tangent angle $\phi$, defined by derivative $\mathrm{d} r / \mathrm{d} z=\operatorname{tg} \phi$. The S- mode profile is considered with continues conjunction with cylinder surfaces of the tube with inner radius $r_{0}$ at the point $A$, and the punch with maximal radius $R_{0}$ at the point $C$. The angle $\phi=0$ at the points $A$ and $C$, and $\phi=$ $\alpha$ at the maximum point $\mathrm{d} \phi / \mathrm{d} z=0$. The profile curvature available should satisfy condition of continues punch-tube contact with positive pressure $p$, defined by solution of differential equilibrium Equation with plastic yield criterion.

Plastic forming of the tube is generated by axial displacement $s$ of the tube rigid part with initial wall thickness $h_{0}$. Displacement $s$ defines deformed tube segment $A B$. If $s=l_{0}$ then point $B$ of the tube edge is coincided with the final punch profile point $C$, where the tube plastic strain increase is stopped. Tube extension process is non-steady at the displacement interval $0 \leq s \leq l_{0}$ with the plastic strain increase and the wall thickness $h$ decrease on the curved tube segment $A B$. If $s \geq l_{0}$ then steady-state extension process begins, with accuracy of friction effect defined by slip of cylindrical tube segment with radius $R_{0}$ on the punch surface.

Tube extension ratio $R_{0} / r_{0}$ is constrained by the limit plastic strain $e_{p}{ }^{*}$ of tension in circular direction $\theta$ of the tube front edge which leads to local increase of the plastic strain followed by fracture of the tube edge. The $e_{p}{ }^{*}$ value is defined by material work hardening behavior [10]. Limit ratio $R_{0} / r_{0}$, defined by the plastic strain $e_{p}{ }^{*}$, is 1.2-1.3 for high plastic steel tube extension by cone punch $[1,4]$. Increase of the metal plasticity by heat of the deformed tube leads to essential increase of extension ratio [4]. In the case of tube extension at elevated temperatures the ideal plastic material model can be used with the yield stress estimation for mean strain rate and temperature values.

Second constraint of the limit tube extension ratio is buckling of thin-walled initial tube induced by compression meridional stress $\sigma_{A}$ at the section $z=0$. Detailed experimental investigations of cylindrical tube buckling are given in Refs $[11,12]$. Approximate estimations of the critical relation $\sigma_{A} / \sigma_{S}$ for the tube extension by cone punch are given in Ref [4]. Critical buckling ratio of the tube can be increased essentially by kinematical constraints of the tube wall in tube extension die design [4].

\section{Stress-Strain Relations}

In the case of thin-walled tube extension deformed material element of the tube middle surface is loaded by membrane principal stresses $\sigma_{1}=\sigma_{\theta}>0$ in circular direc- tion $\theta, \sigma_{2}=\sigma_{\phi}<0$ in meridian direction tangent to the punch profile, and $\sigma_{3}=0$ in normal direction to the punch profile. Generalized Mises yield criterion for the principal stresses in the case of normal anisotropy in direction of the tube wall thickness can be written as follows [7]

$$
\sigma_{\theta}^{2}+\sigma_{\phi}^{2}-\frac{2 a}{1+a}-\sigma_{\theta} \sigma_{\phi}=\sigma_{s}^{2}
$$

Coefficient of normal anisotropy $a$ is ratio of the width to thickness plastic strains defined by axial tension of sheet metal specimen [10]. Material yield stress $\sigma_{\mathrm{s}}$ is defined by accumulated plastic strain $e_{\mathrm{p}}$ using work hardening relation

$$
\sigma_{s}=\sigma_{0}\left(1+C e_{P}^{n}\right)
$$

Plastic flow rule associated with the yield criterion (1) defines increments of the plastic strains in $\phi$ and $\theta$ directions

$$
\mathrm{d} e_{\phi}=c \mathrm{~d} e_{\theta}, \quad c=\frac{(1+a) \sigma_{\phi}-a \sigma_{\theta}}{(1+a) \sigma_{\theta}-a \sigma_{\phi}}
$$

Accumulated effective plastic strain increment $\mathrm{d} e_{p}$ is defined by the $\sigma_{\phi}$ and $\mathrm{d} e_{\phi}$ increments using plastic incompressibility condition

$$
\mathrm{d} e_{p}=\frac{1+a}{\sqrt{1+a+a^{2}}} \sqrt{\mathrm{d} e_{\theta}^{2}+\mathrm{d} e_{\phi}^{2}+\mathrm{d} e_{\theta} \mathrm{d} e_{\phi}}
$$

Substitution relation $\mathrm{d} e_{\theta}=d r / r$ and Equation (3) into Equation (4) defines $\mathrm{d} e_{p}$ as the function of the stress state and circular plastic strain increment

$$
\mathrm{d} e_{p}=\frac{1+a}{\sqrt{1+a+a^{2}}} \sqrt{1+c+c^{2}} \frac{\mathrm{d} r}{r}
$$

Plastic incompressibility condition and Equation (3) define differential relation for the wall thickness versus circular plastic strain increment and stress state coefficient $c$

$$
\frac{\mathrm{d} h}{h}=-(1+c) \frac{\mathrm{d} r}{r}
$$

At the tube edge $B$ (Figure 1) we have $\sigma_{\phi}=0, c=$ $-a /(1+a)$, and wall thickness is found by integration of Equation (6)

$$
h_{B}=h_{0} \exp \left(\frac{1}{1+a} \ln \frac{r_{0}+h_{0} / 2}{r_{B}+h_{B} / 2}\right)
$$

Extension process can be used to form of short ring with initial dimensions $r_{0}, h_{0}, l_{0}$ to final dimensions $R_{0}, h$, $l$, with the wall thickness $h$ defined by Equation (7)

$$
h=h_{0} \exp \left(\frac{1}{1+a} \ln \frac{r_{0}+h_{0} / 2}{R_{0}+h / 2}\right)
$$


and length $l$ defined by constant volume condition

$$
l=l_{0} \frac{h_{0}\left(r_{0}+h_{0} / 2\right)}{h\left(R_{0}+h / 2\right)}
$$

Stress equilibrium Equations considered below will be solved using the yield criterion (1) to write positive circular stress $\sigma_{\theta}$ as the function of meridian stress $\sigma_{\phi}$ and yield stress $\sigma_{s}$

$$
\sigma_{\theta}=\frac{1}{1+a}\left[a \sigma_{\phi}+\sqrt{\left.(1+a)^{2} \sigma_{s}^{2}-(1+2 a) \sigma_{\phi}^{2}\right)}\right]
$$

\section{Tube Stress State}

Stress state of deformed tube is calculated using membrane theory of the rigid-plastic shell with yield criterion (1), work hardening (2), wall thickness variation (6) and Coulomb's friction coefficient $f$ at the punch contact boundary specified by its generator. The shell element equilibrium equation in normal direction to the shell middle surface defines relation of the normal pressure $p$ versus stresses $\sigma_{\varphi}, \sigma_{\theta}$ and the shell curvatures

$$
p=h\left(\frac{\sigma_{\theta}}{R_{2}}-\frac{\sigma_{\phi}}{R_{1}}\right)
$$

If punch generator is specified by the function $r=r(z)$ then curvature radii are defined by the Equations

$$
\begin{gathered}
R_{1}=\left(1+\operatorname{tg}^{2} \phi\right)^{3 / 2}\left(\frac{\mathrm{d}^{2} r}{\mathrm{~d} z_{2}}\right)^{-1}-\frac{h}{2}, \operatorname{tg} \phi=\frac{\mathrm{d} r}{\mathrm{~d} z} \\
R_{2}=\frac{r}{\cos \phi}+\frac{h}{2}
\end{gathered}
$$

From Equation (11) it is follows, that contact pressure $p$ is positive if

$$
\sigma_{\theta}>\sigma_{\phi} R_{2} / R_{1}
$$

If inequality (14) is not satisfied, then the tube is departed from the punch profile, and curved free boundary of the tube is generated. Stress state of extended tube satisfies inequalities $\sigma_{\theta}>0$ and $\sigma_{\phi}<0$. Hence, the inequality (14) is defined by the tube stress state, values and signs of the punch profile radii $R_{1}$ and $R_{2}$.

Tube stress state should satisfy equilibrium Equation in meridian direction to the middle surface, which for the case of variable wall thickness and Coulomb's contact friction is written as follows:

$$
\frac{\mathrm{d} \sigma_{\phi}}{\mathrm{d} r}+\frac{\sigma_{\phi}}{h} \frac{d h}{\mathrm{~d} \phi}+\frac{\sigma_{\phi}-\sigma_{\theta}}{r}-\frac{p f}{h \sin \phi}=0
$$

Substitution Equations (10) and (11) into Equation (15) gives differential Equation for the meridian stress $\sigma_{\phi}$, with specified distributions of the $h$ and $\sigma_{s}$ on the tube middle surface.

If the punch profile is specified by the Equation $r=r$ $(z)$ with continues curvature radii defined by Equations (12) and (13), then integration of Equation (15) can be performed using $z$ variable. In this case Equation (15) can be written in the form:

$\frac{\mathrm{d} \sigma_{\phi}}{\mathrm{d} z}=-\left[\sigma_{\phi}\left(\frac{\operatorname{tg} \phi}{r}+\frac{f}{R_{1} \cos \phi}+\frac{1}{h} \frac{\mathrm{d} h}{\mathrm{~d} z}\right)-\frac{\sigma_{\theta}}{r}(f+\operatorname{tg} \phi)\right](16)$

where $\sigma_{\theta}$ and $R_{1}$ are defined by Equations (10) and (12). If concave and convex punch profile segments are specified by circles radii $r_{1}$ and $r_{2}$, then variable $\phi$ is reasonable for integration of Equation (15). Using relations $\mathrm{d} r=r_{1} \sin \phi \mathrm{d} \phi, \mathrm{d} r=r_{2} \sin \phi \mathrm{d} \phi$ and Equation (11), differential Equation (15) can be written in the forms as Equation (17) on the concave profile segment, and Equation (18) on the convex profile segment.

If the punch profile is cone, conjugated with circles radii $r_{1}$ and $r_{2}$, then Equations (17) and (18) are used on the curved profiles, while the length $l$ of cone generator, inclined at the angle $\alpha$ to the $z$ axis, is used for integration of Equation (15), which takes the form:

$$
\frac{\mathrm{d} \sigma_{\phi}}{\mathrm{d} l}=-\left[\sigma_{\phi}\left(\frac{\sin \alpha}{r}+\frac{1}{h} \frac{\mathrm{d} h}{\mathrm{~d} l}\right)-\frac{\sigma_{\theta}}{r}(\sin \alpha+f \cos \alpha)\right]
$$

Simulation of non-steady tube extension by rigid punch is performed by numerical integration of Equations (16)-(19) by second order Runge method with specified punch profile, and the tube front edge $B$ moving from the point $A$ to the point $C$ (Figure 1). Integrations are performed along the profile from the point $B$, where the boundary conditions are specified

$$
\sigma_{\phi}=0, \sigma_{\theta}=\sigma_{s}\left(e_{p}\right), e_{p}=\ln \left[\left(r_{B}+h_{B} / 2\right) /\left(r_{0}+h_{0} / 2\right)\right],
$$

$$
\begin{gathered}
\frac{\mathrm{d} \sigma_{\phi}}{\mathrm{d} \phi}=-\left[\sigma_{\phi}\left(\frac{\tilde{r}_{1}}{r} \sin \phi+f+\frac{1}{h} \frac{\mathrm{d} h}{\mathrm{~d} \phi}\right)-\sigma_{\theta} \frac{\tilde{r}_{1}}{r}(\sin \phi+f \cos \phi)\right], \tilde{r}_{1}=r_{1}-\frac{h}{2} \\
\frac{\mathrm{d} \sigma_{\phi}}{\mathrm{d} \phi}=-\left[\sigma_{\phi}\left(\frac{\tilde{r}_{2}}{r} \sin \phi-f+\frac{1}{h} \frac{\mathrm{d} h}{\mathrm{~d} \phi}\right)-\sigma_{\theta} \frac{\tilde{r}_{2}}{r}(\sin \phi+f \cos \phi)\right], \tilde{r}_{2}=r_{2}+\frac{h}{2}
\end{gathered}
$$


and thickness $h_{B}$ is defined by Equation (7), to the point $A$, using distributions of the $h, e_{p}$ and $\sigma_{s}$ known for previous position of the point $B$, which is coincided with the point $A$ at the initial process stage. Effective plastic strain increments $d e_{p}$ are calculated from Equations (3) and (5) with material points displacements to the neighbouring nodes of the tube segment $A B$. Summering of the effective plastic strain of material points and calculations of the $\sigma_{\mathrm{s}}$ and $h$ from Equations (2), (6), define distributions of the $\sigma_{\mathrm{s}}$ and $h$ for the next process stage. Calculations of non-steady stages are terminated when the point $B$ is coincided with the point $C$, and steady-state tube extension begins.

Displacement $s$ of initial tube is related with the edge point $B$ position and wall thickness distribution by integral incompressibility condition

$$
s=\frac{1}{h_{0}\left(r_{0}+h_{0} / 2\right)} \int_{A}^{B}\left(\frac{r}{\cos \phi}+h / 2\right) h \mathrm{~d} z
$$

Extension force $P$ versus $s$ is defined by maximum compression stress $\sigma_{A}(s)=-\sigma_{\phi}(s)$ at the point $A$, which is found by integration of Equations (16)-(19) when point $B$ is moving from the point $A$ to the point $C$.

$$
P(s)=2 \pi\left(r_{0}+h_{0} / 2\right) h_{0} \sigma_{A}(s)
$$

FORTRAN programs are written for computer simulation of the tube extension from initial radius $r_{0}$ to the final radius $R_{0}$ with three punch profiles specified on the length $H_{0}$ of the $z$ axis (Figure 1).

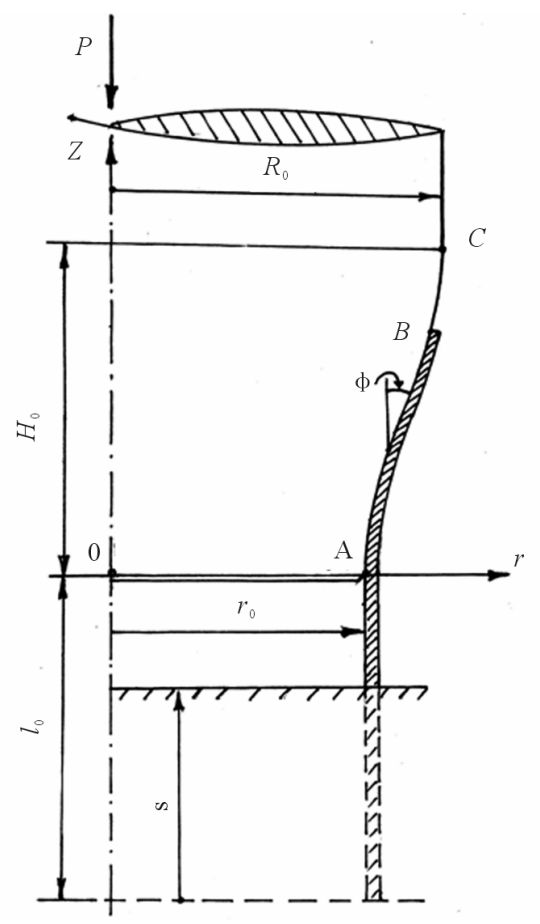

Figure 1. Tube extension by the rigid curved punch.

\section{Punch Profiles}

Cosine profile is specified by the function

$$
r=r_{0}+\frac{R_{0}-r_{0}}{2}\left[1-\cos \left(\pi \frac{z}{H_{0}}\right)\right], 0 \leq z \leq H_{0}
$$

First and second derivatives of the function (23), which defines the tangent angle $\phi$ and curvature radius $R_{1}$ by Equation (12), are as follows

$$
\begin{gathered}
\operatorname{tg} \phi=\frac{\pi}{2} \frac{R_{0}-r_{0}}{H_{0}} \sin \left(\pi \frac{z}{H_{0}}\right) \\
\frac{\mathrm{d}^{2} r}{\mathrm{~d} z^{2}}=\frac{R_{0}-r_{0}}{2}\left(\frac{\pi}{H_{0}}\right)^{2} \cos \left(\pi \frac{z}{H_{0}}\right)
\end{gathered}
$$

Equations (23)-(25) are used for numerical integration of Equation (16) with constant step $\mathrm{d} z=H_{0} /(N-1)$, where $N$ is number of nodes on the punch profile.

Double circular profile is specified by circle radii $r_{1}$ on the concave and $r_{2}$ on the convex segments with tangent angle $\alpha$ at the bend point. The profile parameters $r_{1}$, $r_{2}$ and $\alpha$ satisfy the following relations

$$
\begin{gathered}
\left(r_{1}+r_{2}\right)(1-\cos \alpha)=R_{0}-r_{0} \\
\left(r_{1}+r_{2}\right) \sin \alpha=H_{0}
\end{gathered}
$$

The angle $\alpha$ is found from Equations (26) and (27) in the form

$$
\sin \alpha=\frac{2 d}{1+d^{2}}, d=\frac{R_{0}-r_{0}}{H_{0}}
$$

If radius $r_{1}$ is specified and satisfy the inequality

$$
r_{1}<\frac{H_{0}}{\sin \alpha},
$$

then radius $r_{2}$ can be found from Equation (27), and vice versa. So, double circular punch profile is defined by the parameters $H_{0}, R_{0}, r_{0}$ and $r_{1}$ or $r_{2}$. The profile co-ordinates are specified in parametric form versus tangent angle $\phi$

$$
r=r_{0}+r_{1}(1-\cos \phi), z=r_{1} \sin \phi, 0 \leq \phi \leq \alpha
$$

On the concave segment, and

$$
r=R_{0}-r_{2}(1-\cos \phi), z=H_{0}-r_{2} \sin \phi, \alpha \geq \phi \geq 0
$$

On the convex segment. Equations (30) and (31) are used for numerical integration of Equations (17) and (18) with constant step $\mathrm{d} \phi=\alpha / N-1$, where $N$ is nodes number on the each circular profile.

Cone profile with circular conjunctions is specified by radii $r_{1}$ and $r_{2}$ on the curved concave and convex segments, length $L$ and angle $\alpha$ of the cone segment. The values $r_{1}, r_{2}, L, \alpha$ are related with $H_{0}, R_{0}, r_{0}$ by the Equa- 
tions

$$
\begin{gathered}
\left(r_{1}+r_{2}\right)(1-\cos \alpha)+L \sin \alpha=R_{0}-r_{0} \\
\left(r_{1}+r_{2}\right) \sin \alpha+L \cos \alpha=H_{0}
\end{gathered}
$$

If the angle $\alpha$ satisfy inequality

$$
\frac{\sin \alpha}{1-\cos \alpha}>\frac{H_{0}}{\left(R_{0}-r_{0}\right)},
$$

then $L$ and radii sum are found from Equations (32) and (33)

$$
\begin{gathered}
L=\left(R_{0}-r_{0}\right) \frac{\sin \alpha}{1-\cos \alpha}-H_{0} \\
r_{1}+r_{2}=\frac{H_{0}-L \cos \alpha}{\sin \alpha}
\end{gathered}
$$

So, cone punch profile with circular conjunctions is defined by $H_{0}, R_{0}, r_{0}, \alpha$ and radius $r_{1}$ or $r_{2}$. Co-ordinates of the profile are specified in parametric form versus $\phi$ by Equations (30) and (31) on the curved segments, and versus $l$ on the cone segment

$$
\begin{aligned}
& r=r_{0}+r_{1}(1-\cos \alpha)+l \sin \alpha, z=r_{1} \sin \alpha+l \cos \alpha, \\
& 0 \leq l \leq L
\end{aligned}
$$

Equations (30), (31) are used for numerical integration of Equations (17) and (18) with step $\mathrm{d} \phi$ and nodes number $N_{1}$ on the each curved segment. Equations (37) are used for numerical integration of Equation (19) on the cone segment with step $\mathrm{d} l=L / N_{2}$, where $N_{2}$ is nodes number on the cone profile segment.

\section{Numerical Results}

Numerical results are presented for the tube extension simulations from initial radius $r_{0}=30 \mathrm{~mm}$ with wall thickness $h_{0}=1 \mathrm{~mm}$ to final radius $R_{0}=40 \mathrm{~mm}$ by three curved punch profiles on the length $H_{0}=25 \mathrm{~mm}$. Tube material is low carbon steel with initial yield stress $\sigma_{0}=300 \mathrm{~N} / \mathrm{mm}^{2}$ and work hardening parameters $C=1.27, n=0.672$.

Cosine punch profile, defined by Equation (23), has continues curvature variation with radii $r_{1}=r_{2}=12.67$ $\mathrm{mm}$ at the points $z=0$ and $z=H_{0}$ and tangent angle $\alpha=$ 0.561 at the middle bend point. Double circular profile is specified by radii $r_{1}=15.0 \mathrm{~mm}, r_{2}=21.25 \mathrm{~mm}$ and $\alpha=$ 0.761 at the bend point. Cone profile with circular conjugations is specified by $r_{1}=12.0 \mathrm{~mm}, r_{2}=14.22 \mathrm{~mm}, \alpha=$ 0.5 and $L=14.16 \mathrm{~mm}$ of cone segment.

Force $P$ versus displacement $s$ relations are shown in Figure 2 for three punch profiles with $a=1$ and $f=0.1$, up to the steady state tube extension onset. Relations $P(s)$ are $S$-mode curves with $P_{\max }$ values 28.06, 27.91, 27.83 $\mathrm{kN}$ at the final $s$ values $l_{0}=28.75,30.33,29.94 \mathrm{~mm}$ for (a) cosine, (b) double circular and (c) cone with circular conjunctions punch profiles. Close $P_{\max }$ values are explained by equal length $H_{0}$, extension ratio $R_{0} / r_{0}$ and close curvature radii of the curved punch profile segments.

Distributions of accumulated effective plastic strain $e_{p}$, meridian stress $-\sigma_{\phi} / \sigma_{0}$, contact pressure $\sigma_{s} / \sigma_{0}$, wall thickness $h / h_{0}$ and yield stress $R_{0} / r_{0}$ along deformed tube generator at the final extension stage are shown in Figure 3 for (a) cosine, (b) double circular and (c) cone with circular conjunctions punch profiles. Values $e_{p}, h / h_{0}$, $\sigma_{s} / \sigma_{0}$ and $p / \sigma_{0}$ at the front tube edge $z=H_{0}$, where $\sigma_{\phi}=0$, are defined by the final radius $R_{0}$ for all punch profiles, with $e_{p}=0.282, h / h_{0}=0.868, \sigma_{s} / \sigma_{0}=1.543$ and $p / \sigma_{0}=3.31 \cdot 10^{-2}$. Distributions of $e_{p}, h / h_{0}$, $\sigma_{s} / \sigma_{0}$ are constrained by specified initial and final values, and are close for three punch profiles considered.

Contact pressure $p / \sigma_{0}$ distributions are essentially

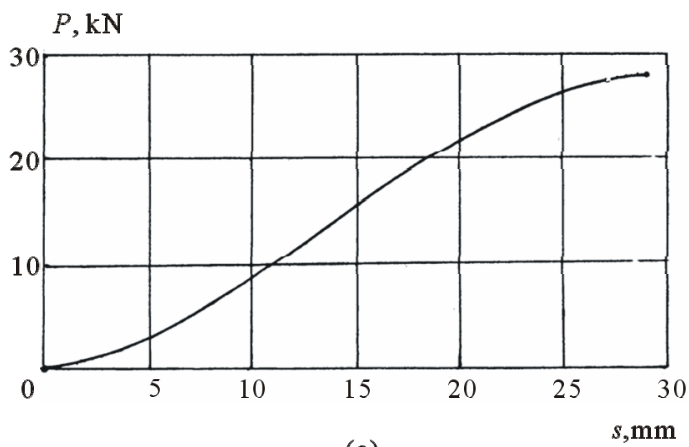

(a)

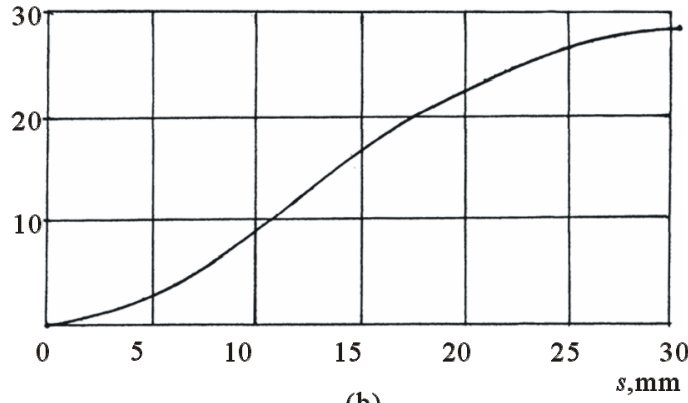

(b)

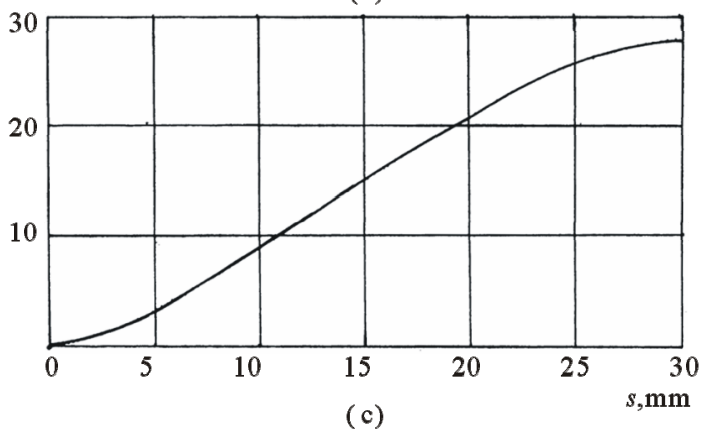

Figure 2. Extension force $P$ versus displacement $s$ for (a) cosine; (b) double circular and (c) cone with circular conjunctions punch profiles. 
different for considered punch profiles. In the case of cosine profile (Figure 3(a)) radius $R_{1}$ on meridian plane and pressure $p / \sigma_{0}$ are continues. Minimal pressure value $2.68 \cdot 10^{-2}$ is at the profile bend point with increase of the pressure to maximal value $6.18 \cdot 10^{-2}$ at the point $z$ $=0$ on concave profile segment. In the case of double circular profile (Figure 3(b)) there is pressure discontinues change from $1.23 \cdot 10^{-2}$ to $4.19 \cdot 10^{-2}$ at the profile bend point, as the result the value and sign discontinuity of radius $R_{1}$ in Equation (11). In the case of cone with circular conjunctions profile (Figure 3(c)) radius $R_{1}$ is discontinues at the points of cone conjunction with con-

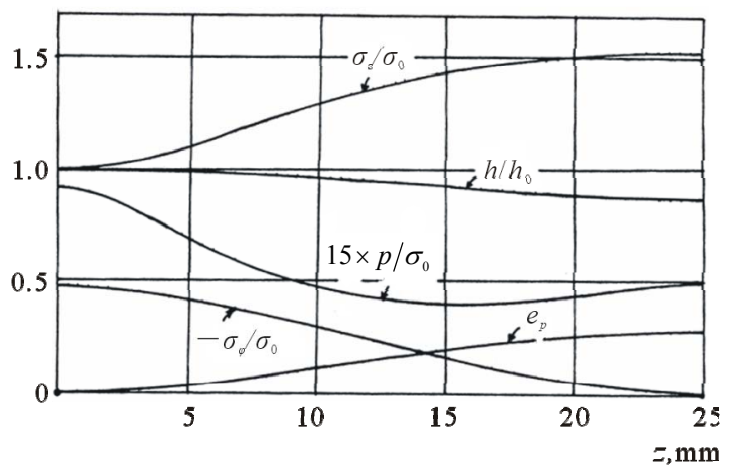

(a)

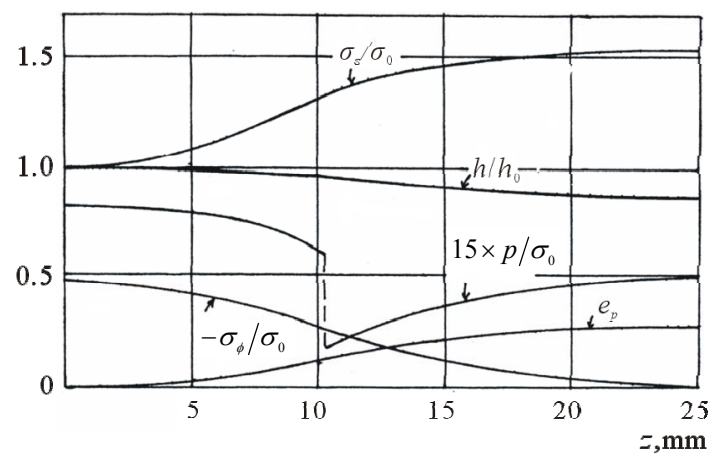

(b)

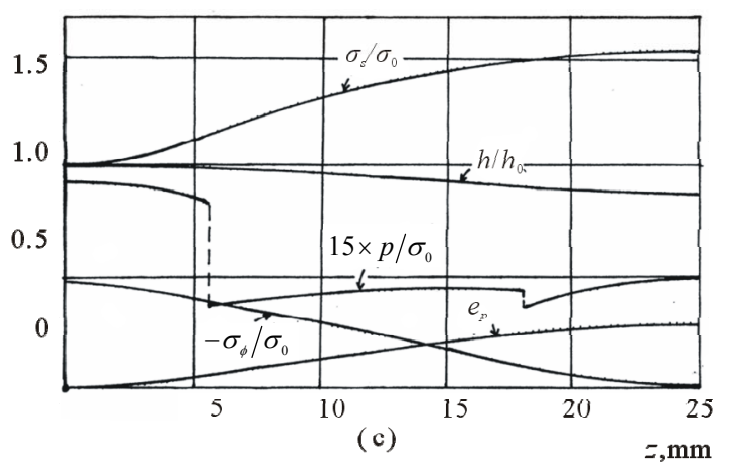

Figure 3. Distributions of plastic strain $\mathrm{e}_{\boldsymbol{p}}$, meridian stress $-\sigma_{\phi} / \sigma_{0}$, wall thickness $h / h_{0}$, yield stress $\sigma_{S} / \sigma_{0}$ and contact pressure $p / \sigma_{0}$ along the tube generator for (a) cosine, (b) double circular and (c) cone with circular conjunctions punch profiles. cave and convex profile segments, where minimal pressures are $2.46 \cdot 10^{-2}$ and $2.45 \cdot 10^{-2}$. Pressure values are decreased on convex profile segments with small compression stress $-\sigma_{\phi}$ and decrease of circular stress $\sigma_{\theta}$, as can see in Equation (11). But modules of negative radius $R_{1}$ are large on convex profile segments, with the result of positive pressure $p$ along all profiles without deviation of deformed tube from the punch contact boundaries.

Distributions of compressive meridian stress $-\sigma_{\phi} / \sigma_{0}$ along profiles are continues increased curves with maximal values $0.488,0.485$ and 0.484 at the point $z=0$ for profiles (a), (b) and (c) accordingly. Effect of different pressure distributions on the $\sigma_{\phi}$ and other variables is negligible, because pressure and friction coefficient in Equations (15)-(19) are small. Increase of anisotropy coefficient $a$ from 1 to 2 leads to increase of minimal wall thickness of the tube front edge, defined by Equation (8), at $4.2 \%$; with decrease of maximal compression stress $-\sigma_{\phi_{\max }}$ and tube extension force $P_{\max }$ at $0.7 \%$ for considered punch profiles.

Friction effect on maximal compression meridian stress $-\sigma_{\phi}$ and extension force $P$ values is given in Table $\mathbf{1}$ for isotropic tube extension to the final radius $R_{0}$ and three punch profiles (a), (b) and (c) considered above. Increase of the friction coefficient $f$ from 0 to 0.15 leads to increase of maximal $-\sigma_{\phi}$ and $P$ values at $42 \%$ for all punch profiles, with a small difference of the profiles lengths.

\section{Experiments}

Experimental verification of the thin-walled tube extension theory has been performed using device for sequential thin-walled rings extension by the punch with S-mode curved profile shown in Figure 4 [13]. First ring 4 with initial dimensions $L_{0}, h_{0}, d_{0}$ is fixed in support 3 groove (left side in Figure 4). Punch 2 pushed by the rod 1 , and first ring is extended up to the middle of the punch

Table 1. Friction and punch profile effects on maximal values of the meridian stress and extension force.

\begin{tabular}{|c|c|c|c|c|}
\hline & \multicolumn{4}{|c|}{$\mathrm{f}$} \\
\hline & 0. & 0.05 & 0.1 & 0.15 \\
\hline \multicolumn{5}{|c|}{ cosine profile } \\
\hline$-\sigma_{\phi} / \sigma_{0}$ & 0.382 & 0.434 & 0.488 & 0.543 \\
\hline$P, \mathrm{kN}$ & 21.95 & 24.98 & 28.06 & 31.19 \\
\hline \multicolumn{5}{|c|}{ double circular profile } \\
\hline$-\sigma_{\phi} / \sigma_{0}$ & 0.377 & 0.431 & 0.485 & 0.541 \\
\hline$P, \mathrm{kN}$ & 21.67 & 24.77 & 27.91 & 31.11 \\
\hline \multicolumn{5}{|c|}{ cone with circular conjunctions profile } \\
\hline$-\sigma_{\phi} / \sigma_{0}$ & 0.378 & 0.431 & 0.484 & 0.538 \\
\hline$P, \mathrm{kN}$ & 21.75 & 24.77 & 27.83 & 30.94 \\
\hline
\end{tabular}


profile (right side in Figure 4). Second ring 5 is fixed in support 3. During second stroke of the punch ring 5 is extended to the middle of the punch profile, and ring 4 is extended to the upper end of the punch. Then ring 6 is fixed in support 3 followed by the punch third stroke, and first ring 4 is pushed from the punch with final dimensions $L, h, d$.

Dimensions $L_{0}, h_{0}, d_{0}$ of specimens for extension by the punch were obtained by the rings reduction through the matrix with S-mode profile shown in Figure 5 [14] and used for verification of the thin-walled tube reduction model [9]. First ring 4 with initial dimensions $L_{0}, h_{0}$, $d_{0}$ is fixed in cylindrical part of the matrix with diameter $d_{0}$ and pushed to the middle of the matrix profile by the punch 1 . Second ring 5 is fixed in the matrix and pushed to the middle of the matrix by second stroke of the punch, while first ring is pushed to the end of the matrix profile. Finally, ring 6 is fixed in the matrix followed by its pushing to the middle of the matrix, ring 5 is pushed to the end of the matrix during third punch stroke, and first ring 4 is pushed out of the matrix with final dimensions $L$, $h, d$.

Extension of reduced rings by the curved punch was used to increase plastic strain and properties of the rings. Samples for reduction and extension experiments with dimensions $d_{0}=39.9 \mathrm{~mm}, h_{0}=1.98 \mathrm{~mm}, L_{0}=17.3 \mathrm{~mm}$

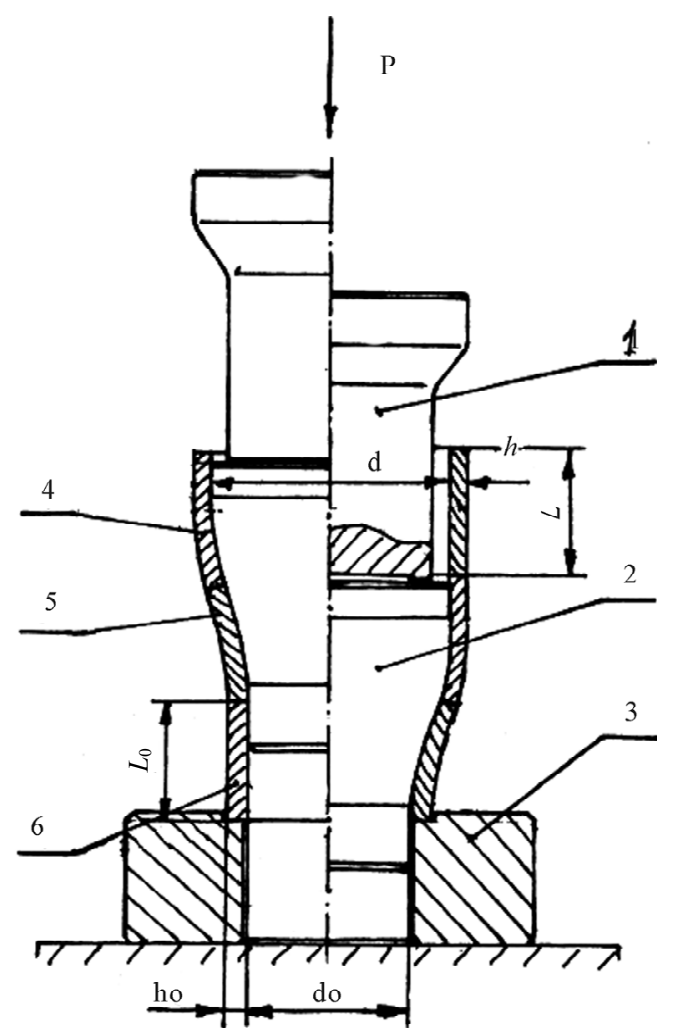

Figure 4. Device for sequential rings extension by curved punch. 1-push rod, 2-punch, 3-support, 4-6-extended rings.

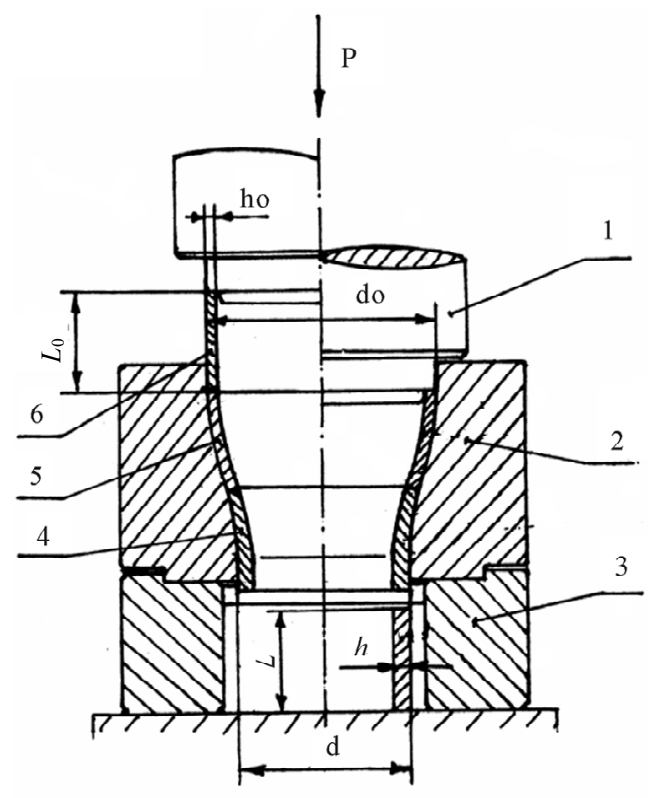

Figure 5. Device for sequential rings reduction by curved matrix. 1-punch, 2-matrix, 3-support, 4-6-reduced rings.

(Figure 5) were turned from hot rolled tube of carbon steel St 3 (Russian metallurgy standard). Work hardening curve $\sigma_{s}\left(e_{p}\right)$ was found by compression tests of short ring specimens, turned from the tube, by lubricated smooth flat dies. Material parameters of the approximation (2) are $\sigma_{0}=320 \mathrm{H} / \mathrm{mm}^{2}, C=1.8$ and $n=0.4$. Isotropic material is assumed, with $a=1$ in Equations (3)-(10).

Cosine profiles (23) were used for the matrix and punch manufactured on machine tool with digital control program, followed by the heat treatment for specified hardness. Profile (23) parameters are $R_{0}=18 \mathrm{~mm}, r_{0}=$ $13.74 \mathrm{~mm}, H_{0}=30 \mathrm{~mm}$ for the punch (Figure 4), and $R_{0}$ $=20 \mathrm{~mm}, r_{0}=16 \mathrm{~mm}, H_{0}=30 \mathrm{~mm}$ for the matrix (Figure 5). Reduction and extension experiments were performed on standard hydraulic testing machine with bough the force and displacement control. Ring specimens, punch and matrix profiles were lubricated by oil-graphite suspension. Coulomb's friction coefficient $f$ for the tool roughness with lubrication was assumed in the range 0.05-0.08.

Comparison of predicted relations $P(s)$ for the rings reduction by curved cosine matrix [9] with experimental data is shown in Figure 6. Non-steady and steady state experimental data are reasonable correlated with predicted relations in the range of possible Coulomb's friction coefficient values. Predicted final ring dimensions after reduction $[9,14] h=2.26 \mathrm{~mm}, L=19.5 \mathrm{~mm}$ are good correlated with measured dimensions $h=2.3 \mathrm{~mm}$, $L=19.3 \mathrm{~mm}$ of the reduced rings.

Device for the rings extension by the curved cosine 
punch (Figure 4) has been manufactured several months later after rings reduction device with the result of reduced work hardening effect relaxation. Comparison of predicted relations $P(s)$ for the reduced rings extension by the curved cosine punch of the present model with experimental data is shown in Figure 7. Non-steady experimental data are good correlated with the model in the range $0.05-0.08$ of the friction coefficient $f$. First ring extension to the middle of the punch profile is close to predicted curve with $f=0.08$, while further two rings extension to the end of the punch profile is close to predicted curve with $f=0.05$. Predicted final ring dimensions after extension $h=2 \mathrm{~mm}, L=17.24 \mathrm{~mm}$ are good correlated with measured dimensions $h=1.95 \mathrm{~mm}, L=$ $17.3 \mathrm{~mm}$ of the final rings.

\section{Conclusions}

Model of thin-walled tube extension by curved rigid punch based on membrane rigid-plastic theory are developed by numerical solution of differential Equations along specified curved punch generator with consideration of material normal anisotropy, work hardening, contact friction and wall thickness variation, defined by generalized Mises flow rule.

FORTRAN programs of the model predict extension force versus displacement and distributions of effective plastic strain, yield stress, meridian stress and contact pressure along the tube generator at specified punch or tube displacement up to steady state process beginning. Positive contact pressure, defined by specified $S$-mode punch profiles, is essential condition for the tube forming without deviation from contact boundary with the punch.

Numerical examples of mild steel tube extension by $S$-mode cosine, double circular and cone with circular conjunctions punch profiles for extension ratio $R_{0} / r_{0}=$

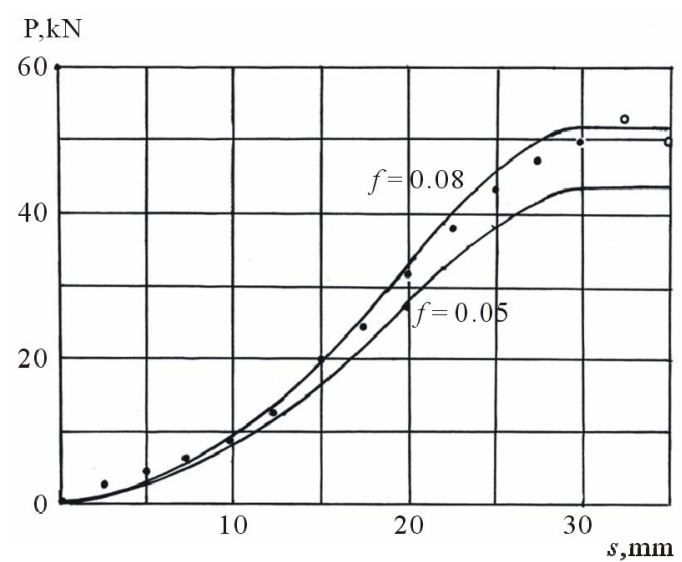

Figure 6. Predicted (solid lines) and experimental (•-non-steady, ○-steady state) relations $P(s)$ for thin-walled rings reduction by curved cosine matrix.

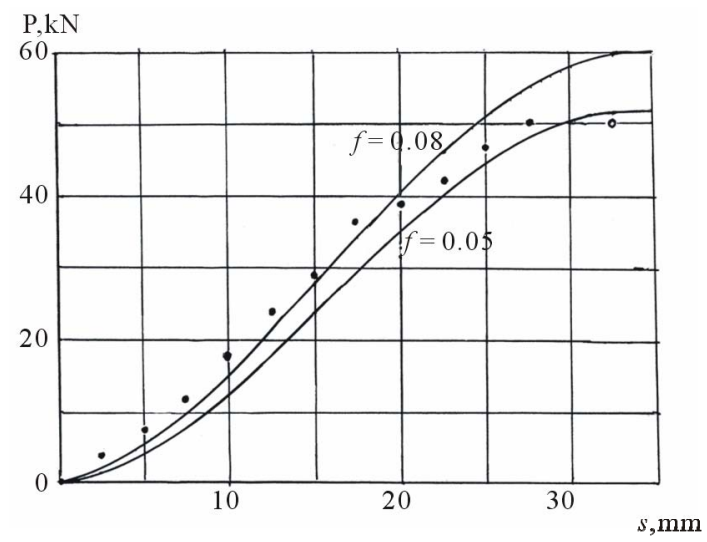

Figure 7. Predicted (solid lines) and experimental (•non-steady, ○-steady state ) relations $P(s)$ for thin-walled rings extension by curved cosine punch.

1.3 with normal anisotropy variation from 1 to 2 show increase of minimal thickness of the tube front edge at $4.2 \%$ with decrease of maximal extension force at $0.7 \%$. Increase of the friction coefficient from 0 to 0.15 leads to drastic growth of extension force and maximal compression meridian stress, which can be constrained by the tube buckling.

Models of thin-walled tube extension by curved punch and thin-walled tube reduction by curved matrix [9] are used in patents $[13,14]$ for thin-walled rings plastic forming. Predicted force-displacement relations and dimensions of thin-walled carbon steel rings forming by lubricated matrix and punch are reasonable correlated with experimental data.

\section{References}

[1] V. P. Romanovsky, "Cold Stamping Handbook," Mashinostroenie, Leningrad, 1979.

[2] E. A. Popov, "Basis of Sheet Stamping Theory," Mashinostroenie, Moscow, 1977.

[3] E. A Popov, V. G. Kovalev and I. N. Shubin, "Technology and Automation of Sheet Stamping," Baumann University Press, Moscow, 2003.

[4] Ju. A. Averkiev and A. Ju. Averkiev, "Cold Stamping Technology," Mashinostroenie, Moscow, 1989.

[5] R. I. Nepershin, "Simulation of Thin-Walled Axisymmetrical Shell Drawing with Flat Flange," KuznechnoShtampovochnoe Proizvodstvo: Obrabotka Materialov Davleniem, No. 6, 2008, pp. 31-36.

[6] R. I. Nepershin, "Simulation of thin-Walled Axisymmetrical Shell Drawing with Flat Flange (Continuation)," Kuznechno-Shtampovochnoe Proizvodstvo: Obrabotka Materialov Davleniem, No.7, 2008, pp. 34-40.

[7] R. I. Nepershin, "Simulation of Thin-Walled Axisymmetrical Shell Drawing by Complex Form Punch with Normal Anisotropy and work hardening of Workpiece Mate- 
rial Consideration," Kuznechno-Shtampovochnoe Proizvodstvo: Obrabotka Materialov Davleniem, No. 3, 2009 , pp. 33-37.

[8] R. I. Nepershin, “Thin-Walled Conical Shell Drawing from a Plane Blank," Mechanics of Solids, Vol. 45. No. 1, 2010, pp. 111-122.

[9] R. I. Nepershin, "Pressing of Thin-Walled Tube by a Curvilinear Matrix," Journal of Machinery Manufacture and Reliability, Vol. 38, No. 3, 2010, pp. 263-269.

[10] A. D. Tomlenov, "Theory of Metals Plastic Deformation," Metallurgy, Moscow, 1972.

[11] K. R. F. Andrews, G. L. England and E. Ghani, "Classification of the Axial Collapse of Cylindrical Tubes under Quasi-Static Loading," International Journal of $\mathrm{Me}$ - chanical Sciences, Vol. 25, No. 9-10, 1983, pp. 687-696. doi:10.1016/0020-7403(83)90076-0

[12] A. G. Mamalis and W. Johnson, "The Quasi-Static Crumpling of Thin-Walled Circular Cylinders and Frusta under Axial Compression," International Journal of Mechanical Sciences, Vol. 25, No. 9-10, 1983, pp. 713-732. doi:10.1016/0020-7403(83)90078-4

[13] R. I. Nepershin, "Device for Extension of Thin-Walled Cylindrical Rings," Russian Federation Patent, No. 85377, Registered in 10 August 2009.

[14] R. I. Nepershin, "Device for reduction of Thin-Walled Cylindrical Rings," Russian Federation Patent No. 95280, Registered in 27 June 2010.

\section{Notation}

$r_{\mathrm{o}}$
$R_{\mathrm{o}}$
$h_{\mathrm{o}}$
$h$
$s$
$l_{\mathrm{o}}$
$f$
$r, z, \theta$
$H_{\mathrm{o}}$
$P$
$R_{1}$
$R_{2}$
$\phi$
$\sigma_{0}$
$e_{\mathrm{p}}$
$\sigma_{\mathrm{s}}$
$a$
$\sigma_{\theta}$
$\sigma_{1}, \sigma_{2}$
$\sigma_{\theta}$

$r_{\mathrm{o}}$

$H_{\mathrm{o}}$

$\sigma_{1}, \sigma_{2}$

$\sigma_{\theta}$

$\tau$ minimal punch radius and initial tube inner radius $(\mathrm{mm})$

maximal punch radius and final tube inner radius $(\mathrm{mm})$

initial thickness of the tube wall $(\mathrm{mm})$

variable thickness of the tube wall $(\mathrm{mm})$

tube displacement relative the fixed punch $(\mathrm{mm})$

final tube displacement at the end of non-steady process $(\mathrm{mm})$

Coulomb's friction coefficient

cylindrical co-ordinates

length of curved punch profile along the $\mathrm{z}$ axis $(\mathrm{mm})$

tube extension force $(\mathrm{kN})$

curvature radius of the tube middle surface on meridian plane $(\mathrm{mm})$

curvature radius of the tube middle surface on normal plane $(\mathrm{mm})$

tangent angle of the punch profile and tube middle surface with the $z$ axis

initial yield stress of the tube material $\left(\mathrm{H} / \mathrm{mm}^{2}\right)$

accumulated effective plastic strain

yield stress of the tube material defined by $e_{\mathrm{p}}\left(\mathrm{H} / \mathrm{mm}^{2}\right)$

normal anisotropy parameter-relation of the width to thickness plastic strains measured during tension test of the sheet specimen

membrane principal stresses

meridian stress of the tube middle surface element

circular stress of the tube middle surface element

normal pressure on the punch profile

friction stress on the punch profile 